\title{
Tamizaje de enfermedades infecciosas en bancos de sangre, Colombia, 1995
}

\author{
Mauricio Beltrán ${ }^{1}$, Jorge Raad ${ }^{2}$, Maribel Ayala ${ }^{1}$, Regina Ching ${ }^{1}$
}

\section{Resumen}

Con el fin de estimar la prevalencia de las enfermedades transmitidas por la sangre, mediante las pruebas de tamizaje en los donantes de sangre de los bancos del país, pertenecientes a la red nacional, se analizó la información enviada por los bancos de sangre en 1995 a la Coordinación Nacional de Bancos de Sangre en el Instituto Nacional de Salud.

Se definieron como variables de estudio las siguientes: unidades de sangre obtenidas, unidades de sangre analizadas y unidades de sangre reactivas para anticuerpos contra el virus de inmunodeficiencia humana tipos 1 y 2 (VIH 1/2), el virus de hepatitis $C$ (VHC), el virus linfotrópico humano (HTLV), Treponema pallidum (sífilis) y Trypanosoma cruzi (enfermedad de Chagas) y reactivas para el antígeno de superficie de hepatitis $B$ (HBsAg); para el análisis, se utilizó el programa Epi-Info 5.1.

Durante 1995, en Colombia se recolectaron 370.867 unidades de sangre en 172 bancos. En promedio, el porcentaje de sangre analizada por estos bancos fue superior a $99 \%$ para marcadores como $\mathrm{VIH} \mathrm{1/2,} \mathrm{HBsAg,} \mathrm{VHC} \mathrm{y} \mathrm{Treponema} \mathrm{pallidum;} \mathrm{para} \mathrm{otros} \mathrm{marcadores}$ como $T$. cruzi y HTLV $1 / 2$ fue de $47,9 \%$ y $15,1 \%$, respectivamente.

Del total de unidades de sangre analizadas por marcador, $0,3 \%$ presentó reactividad para $\mathrm{VIH}-1 / 2$; para hepatitis B virus, $0,9 \%$; para hepatitis $\mathrm{C}$ virus, $1,0 \%$; para $T$. cruzi, $1,2 \%$; para T. pallidum, $1,4 \%$ y para HTLV, $1,2 \%$. Estos porcentajes corresponden a los promedios nacionales de unidades de sangre reactiva en los bancos del país.

Los bancos de sangre de algunas seccionales informaron valores hasta cuatro veces superiores al promedio nacional para los marcadores anti-T. cruzi y HBsAg.

La disponibilidad de sangre en el país es de aproximadamente 11 unidades por cada mil habitantes. El tamizaje en las unidades de sangre detectó $6,08 \%$ de unidades de sangre reactivas; es decir que previno, por lo menos, la difusión de igual número de infecciones por cada 100 posibles transfusiones. El análisis de la información de los bancos de sangre en las seccionales del país, refleja la necesidad de mejorar los procesos de selección de donantes y brinda una aproximación acerca del comportamiento de estas patologías en la población general.

${ }_{1}$ Grupo de Bancos de Sangre, Subdirección de Epidemiología y LNR, Santa Fe de Bogotá.

2 Subdirección de Epidemiología y LNR, Santa Fe de Bogotá. 


\title{
Summary
}

As infectious diseases are the major cause of morbidity in Colombia, the objective of this study was to estimate the prevalence of blood transmitted diseases by screening tests in blood donors. Thus, the data collected from the National Blood-Bank Network was analysed.

\begin{abstract}
All records received during 1995 at the National Blood Banks' Coordination Centre from local blood banks all over the country were reviewed. Some variables such as: number of blood units obtained, number of blood units analysed and number of blood units reactive to HIV $1 / 2$, hepatitis $B$ and $C$ viruses, human trophic lymphocyte virus, T. pallidumand $T$. cruzi were analysed. Epi Info 5.0. was used to analyse the data collected.
\end{abstract}

370,815 blood units were obtained from 172 blood banks. On average, the percentage of blood units analysed for markers such as HIV, hepatitis $B$ and $C$ viruses and $T$. pallidum was higher than $99 \%$; for other markers such as T. cruzi and HTLV $1 / 2$ the percentages were $47 \%$ and $15 \%$, respectively.

Of the total blood units analysed, $0.3 \%$ were reactive to HIV $-1 / 2,1.2 \%$ to HTLV, $1.0 \%$ to hepatitis $C$ virus, $0.9 \%$ to hepatitis $B$ virus, $1.2 \%$ to $T$. cruzi and $1.4 \%$ to T. pallidum. These percentages correspond to national averages from reactive blood units analysed in all banks. Some blood banks reported values for anti-T. cruzi and HBsAg 4 times higher than the national average.

The availability of blood units in this country is approximately 11 units per 1,000 inhabitants. $6.08 \%$ of screened blood units were reactive to any of the infectious agents described above. Blood screening was shown to be helpful in the prevention of infectious agents' transmission, at least, in the same number of infections per 100 possible transfusions.

This analysis showed the need for better donor selection procedures at local blood banks in order to decrease infectious agents' transmission through blood transfusion.

Los bancos de sangre en Colombia iniciaron el control de enfermedades trasmitidas por la sangre en la década de 1950-1960 con la prueba de cardiolipina VDRL que detecta anticuerpos antiTreponema pallidum (sífilis); posteriormente, en 1978, se adicionó el rastreo de antígeno de hepatitis B (HBsAg). El Ministerio de Salud formalizó estos procedimientos mediante el decreto 616 del 11 de marzo de 1981. En 1982, con la aparición del síndrome de inmunodeficiencia adquirida (SIDA) y debido a que la transfusión se identificó como una de las principales vías de transmisión, se implementó la detección del VIH a finales de la década (1). Hoy se estima que la probabilidad de que una persona que reciba una unidad de sangre contaminada se infecte con el VIH es superior a $95 \%$, lo cual convierte a la transfusión como la vía más eficiente de transmisión del virus (2).
Los decretos 559 de 1991 y 1571 de 1993, expedidos por el Ministerio de Salud, establecieron las pruebas necesarias que se deben realizar en las unidades de sangre definiéndolas como obligatorias: anticuerpos anti$\mathrm{VIH}$ tipos 1 y 2, HBsAg, anticuerpos anti-virus hepatitis $\mathrm{C}(\mathrm{VCH})$, anticuerpos anti-Treponema pallidum y, finalmente, mediante la resolución 01738 de mayo de 1995 se adoptó la prueba de anticuerpos anti-Trypanosoma cruzi, para la enfermedad de Chagas (3).

En 1993, se creó la Coordinación Nacional de Bancos de Sangre con sede en el Instituto Nacional de Salud $(4,5)$. Esta coordinación ha venido recopilando y analizando la información pertinente de todos los bancos de sangre públicos y privados del país y estableciendo indicadores que permitan conocer la realidad nacional, regional y seccional en cuanto a recolección, 
fraccionamiento y disponibilidad de la sangre, así como la distribución de las enfermedades sometidas a control en la sangre. Esta información ha servido como apoyo para tomar decisiones en los niveles técnico, investigativo y administrativo y ha mejorado la calidad de la sangre que se transfunde en el país.

\section{Materiales y métodos}

Se realizó un análisis descriptivo de tipo retrospectivo con la información de todos los bancos de sangre del país que informaron a esta coordinación.

Durante 1995, se recopiló mensualmente la información de 172 de los 174 bancos de sangre del país, mediante el formulario establecido por el Ministerio de Salud a través de la Coordinación Nacional de la Red de Bancos de Sangre. No se obtuvieron datos de las seccionales de Chocó y Vaupés.

La información fue consolidada por las coordinaciones seccionales de los bancos de sangre y, luego, enviada a la Coordinación Nacional. Se realizó un análisis univariado utilizando medidas de tendencia central y de dispersión con las bases de datos en Epilnfo 5.1 y Excel. Se utilizaron como variables las unidades de sangre obtenidas (USO), las unidades de sangre analizadas y las reactivas para anticuerpos anti-VIH, tipo 1 y 2, HBsAg, anticuerpos anti-VHC, anticuerpos anti-Treponema pallidum, anticuerpos anti-Trypanosoma cruzi y anticuerpos anti-virus linfotrópico humano HTLV 1 y 2.

\section{Resultados}

En 1995, los 172 bancos de sangre del país que enviaron su información, recolectaron por donación 370.867 unidades de sangre (cuadro 1).

Se halló que, de 370.364 unidades de sangre analizadas o tamizadas para $\mathrm{VIH}, 3 \%$ eran reactivas para $\mathrm{HBsAg}$; se analizaron 369.811 y fueron reactivas $0,9 \%$; para $\mathrm{VHC}$, se analizaron 367891 resultando reactivas $1.0 \%$; para $T$. pallidum, se analizaron 368.213 unidades de sangre y fueron reactivas $1,4 \%$; para $T$. cruzi, de 177.929 unidades de sangre tamizadas resultaron reactivas el $1,2 \%$ y, finalmente, de 55.949 unidades analizadas para HTLV 1 y 2 fueron reactivas $1,2 \%$. Estas cifras representan el promedio nacional de unidades de sangre que resultaron reactivas para los anteriores marcadores en todos los bancos de sangre del país.

En promedio, el porcentaje de sangre analizada por los bancos de sangre fue superior a $99 \%$ para marcadores como: $\mathrm{VIH}, \mathrm{HBsAg}, \mathrm{VHC}$ y $T$. pallidum; para otros marcadores como T. cruziy HTLV $1 / 2$ fueron de 45,9 y $15,1 \%$. Se observó un incremento notable en el tamizaje de $T$. cruzi ya que sólo hasta este año se estableció como prueba obligatoria.

El análisis de tamizaje por seccional mostró que los bancos de sangre de 24 de las 33 seccionales (72\%) informaron como tamizadas para $\mathrm{VIH}$ todas las unidades de sangre obtenidas; los de 25 de las seccionales $(75 \%)$ lo hicieron para HBsAg; los de $23(69 \%)$ para anticuerpos contra hepatitis $\mathrm{C}$ y anti-T. pallidum; en los bancos de sangre de tres de las seccionales se analizaron todas las unidades de sangre para HTLV 1/2. Solamente los bancos de sangre de tres de las 33 seccionales $(1 \%)$ realizaron todas las pruebas establecidas como obligatorias al $100 \%$ de las unidades de sangre recolectadas.

Se observaron valores de reactividad superiores al promedio nacional para anti-VIH en $12(36,3 \%)$ de las 33 seccionales; algunas de éstas fueron: Guaviare 1,5\%, Magdalena 1,3\% y Vichada 1,0\%. Del total de las seccionales, $18(54.5 \%)$ presentaron valores superiores a la reactividad promedio nacional para el antígeno de superficie de hepatitis $B$; entre éstas, Vichada $8,4 \%$, Magdalena 4,3\%, Guaviare 3,0\% y Cesar 2,6\%. Ocho seccionales $(24,2 \%)$ informaron reactividades superiores al valor promedio nacional para anticuerpos anti-virus de hepatitis C y, únicamente, Guaviare 2,4\% presentó valores dos veces superiores (cuadro 1 ).

Para anticuerpos anti-T. pallidum, se observó que 18 de las 33 seccionales presentaron una reactividad superior al promedio nacional, destacándose Guaviare 5,0\%, Tolima 3,3\% y Magdalena 2,3\%. Los porcentajes más elevados de reactividad para anti-T.cruzi correspondieron a las seccionales tradicionalmente endémicas para la enfermedad como Casanare $8,9 \%$, 
Cuadro 1. Porcentaje de serorreactividad por seccional, Colombia 1995.

\begin{tabular}{|c|c|c|c|c|c|c|c|}
\hline Seccional & USO $^{\star}$ & VIH & $\mathrm{HBsAg}$ & $\mathrm{VCH}$ & T.pallidum & T.cruzi & HTLV \\
\hline Amazonas & 515 & 0,2 & 2,5 & 1,3 & 1,6 & 0,0 & $\mathrm{~N}, \mathrm{R}$ \\
\hline Antioquia & 8.034 & 0,3 & 1,0 & 1,1 & 1,7 & 0,5 & $\mathrm{~N}, \mathrm{R}$ \\
\hline Arauca & 1.038 & 0,4 & 1,9 & 1,3 & 2,3 & 3,9 & $\mathrm{~N}, \mathrm{R}$ \\
\hline Atlántico & 13.114 & 0,3 & 0,9 & 0,6 & 0,3 & 0,0 & $\mathrm{~N}, \mathrm{R}$ \\
\hline * Bogotá & 122.321 & 0,4 & 0,8 & 1,2 & 1,3 & 1,4 & $\mathrm{~N}, \mathrm{R}$ \\
\hline Bolívar & 7.311 & 0,4 & 0,6 & 0,6 & 0,7 & 0,2 & $\mathrm{~N}, \mathrm{R}$ \\
\hline Boyacá & 7.293 & 0,3 & 0,9 & 0,8 & 1,7 & 1,0 & $\mathrm{~N}, \mathrm{R}$ \\
\hline Caldas & 14.264 & 0,1 & 0,6 & 1,0 & 1,3 & 0,6 & $\mathrm{~N}, \mathrm{R}$ \\
\hline *Cali & 41.783 & 0,2 & 0,4 & 1,3 & 1,8 & 0,9 & 1,6 \\
\hline Caquetá & 2.132 & 0,1 & 0,8 & 0,8 & 2,1 & 1,0 & $\mathrm{~N}, \mathrm{R}$ \\
\hline Casanare & 688 & 0,0 & 2,5 & 0,6 & 1,7 & 8,9 & $\mathrm{~N}, \mathrm{R}$ \\
\hline Cauca & 4.626 & 0,2 & 0,9 & 0,3 & 0,2 & 0,1 & 0,1 \\
\hline Cesar & 4.355 & 0,7 & 2,6 & 0,7 & 2,3 & 2,2 & $\mathrm{~N}, \mathrm{R}$ \\
\hline Córdoba & 5.841 & 0,2 & 0,7 & 0,4 & 2,0 & 0,8 & $\mathrm{~N}, \mathrm{R}$ \\
\hline Cundinamarca & 8.067 & 0,5 & 1,8 & 0,9 & 1,5 & 1,2 & $\mathrm{~N}, \mathrm{R}$ \\
\hline Guanía & 108 & 0,0 & 0,0 & 0,0 & 0,9 & 0,0 & $\mathrm{~N}, \mathrm{R}$ \\
\hline La Guajira & 1.841 & 0,5 & 2,1 & 0,8 & 2,0 & 1,0 & $\mathrm{~N}, \mathrm{R}$ \\
\hline Guaviare & 269 & 1,7 & 3,3 & 2,5 & 5,8 & 1,7 & $\mathrm{~N}, \mathrm{R}$ \\
\hline Huila & 5.066 & 0,1 & 0,5 & 0,4 & 0,5 & 1,7 & $N, R$ \\
\hline Magdalena & 3.414 & 1,3 & 4,3 & 0,6 & 2,3 & 0,0 & $\mathrm{~N}, \mathrm{R}$ \\
\hline *Medellín & 46.427 & 0,3 & 0,5 & 1,0 & 0,8 & 0,9 & $\mathrm{~N}, \mathrm{R}$ \\
\hline Meta & 5.029 & 0,2 & 1,5 & 0,4 & 0,8 & 1,8 & $\mathrm{~N}, \mathrm{R}$ \\
\hline Nariño & 4.606 & 0,1 & 1,1 & 0,3 & 1,2 & 0,1 & 0,2 \\
\hline Norte de Santander & r 8.742 & 0,2 & 3,9 & 0,9 & 1,8 & 3,5 & $\mathrm{~N}, \mathrm{R}$ \\
\hline Putumayo & 487 & 0,6 & 0,6 & 0,0 & 0,8 & 0,0 & $\mathrm{~N}, \mathrm{R}$ \\
\hline Quindío & 6.117 & 0,5 & 0,5 & 0,6 & 2,1 & 0,3 & $\mathrm{~N}, \mathrm{R}$ \\
\hline Risaralda & 8.418 & 0,2 & 0,4 & 0,7 & 2,7 & 0,3 & $\mathrm{~N}, \mathrm{R}$ \\
\hline San Andres & 308 & 0,6 & 1,9 & 1,3 & 0,0 & 0,0 & $\mathrm{~N}, \mathrm{R}$ \\
\hline Santander & 18.161 & 0,2 & 1,2 & 0,8 & 1,7 & 2,0 & $\mathrm{~N}, \mathrm{R}$ \\
\hline Sucre & 2.121 & 0,2 & 1,0 & 0,3 & 0,7 & 0,0 & $\mathrm{~N}, \mathrm{R}$ \\
\hline Tolima & 9.601 & 0,3 & 1,1 & 1,2 & 3,3 & 0,8 & $\mathrm{~N}, \mathrm{R}$ \\
\hline Valle & 8.695 & 0,2 & 1,0 & 0,5 & 2,0 & 0,3 & 0,5 \\
\hline Vichada & 104 & 1,0 & 8,4 & 0,0 & 0,0 & 0,0 & $\mathrm{~N}, \mathrm{R}$ \\
\hline TOTAL & 370.867 & 0,3 & 0,9 & 1,0 & 1,4 & 1,2 & 1,2 \\
\hline
\end{tabular}

Las ciudades capitales informan independientemente USO: unidades de sangre obtenidas NR: no realizan tamizaje.

Arauca 3,9\%, Norte de Santander 3,5\%, Cesar $2,2 \%$ y Santander $2,0 \%$. Finalmente, en el tamizaje de anti-HTLV, que se realiza exclusivamente en cuatro seccionales, solamente Cali notificó $1,6 \%$ de reactividad, siendo superior al promedio nacional haliado para este marcador (cuadro 1).
Los bancos de Santa Fe de Bogotá y de las seccionales de Valle y Antioquia que recolectan el $56 \%$ de las unidades de sangre del país, presentaron valores de reactividad cercanos al promedio nacional observado para todos los marcadores, a diferencia de los bancos de sangre de otras seccionales que recolectan un porcentaje 
muy bajo y donde se observaron los valores de reactividad más altos para todos los marcadores (cuadro 1).

Del total de unidades de sangre tamizadas, se descartó el $6,08 \%$ por causa de reactividad a cualquiera de las pruebas realizadas, principalmente por reactividad a anti-Treponema pallidum, $\mathrm{HBsAg}$ $y$ anti-hepatitis $C$ (figura 1).

\section{Discusión}

El desarrollo de la red de bancos de sangre a nivel nacional dentro de las instituciones hospitalarias, hace que gran parte de la sangre que se recolecta en nuestro país sea de donantes tipo intrafamiliar. Esta estructura es causa, en gran parte, de la falta de programas formales de fomento y promoción de la donación altruísta, de la baja disponibilidad de unidades de sangre -11 por cada mil habitantes comparada con 40 a 50 por cada mil en países desarrollados- y del número elevado de unidades de sangre reactiva que puede incrementar el riesgo de transmitir infecciones por vía transfusional. El análisis muestra que, en promedio, 2 a 3 de cada 1.000 unidades de sangre resultan reactivas para $\mathrm{VIH}$ y entre 8 y 12 unidades de cada 1000 para los demás microorganismos sometidos a vigilancia, promedios varias veces mayores que los observados en países como Inglaterra y Estados Unidos (4, 9).

Sin embargo, es probable que se hayan evitado 6,08 nuevas infecciones por cada 100 unidades tamizadas, al detectar la presencia de cualquiera de estos marcadores en la sangre y teniendo en cuenta que cada unidad de sangre está siendo transfundida, por lo menos, a un individuo receptor.

\section{Conclusiones}

Tres años después de la vigencia del decreto que reglamentó lo relacionado con la extracción, procesamiento y uso de la sangre, se observa un notable desarrollo y mejoramiento de la calidad de la misma, en la existencia de información documentada de los diferentes procesos de bancos de sangre y lo relacionado con el análisis serológico de las unidades de sangre obtenidas en el país.

La vigilancia permanente de las actividades de banco de sangre muestra que las reactividades obtenidas
Figura 1. Total de unidades de sangre descartada por reactividad a los diferentes marcadores, Colombia, 1995.

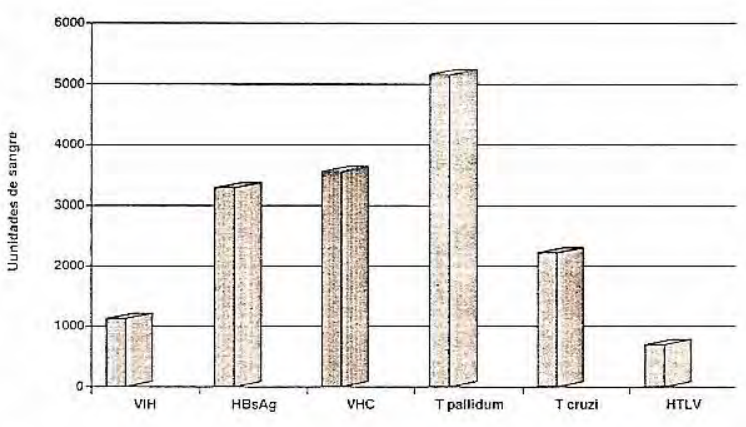

-por el tamizaje de los agentes transmitidos por la sangre, pueden servir como indicador de la tendencia de éstos en la población general y ser un importante apoyo a la salud pública (4, 6). También, la actividad de tamizaje en los bancos de sangre ha evitado un gran número de posibles infecciones transmitidas por la sangre, como se muestra para el período informado. La meta inmediata es el tamizaje de todas las unidades de sangre obtenidas en el país, para los marcadores aprobados, de tal forma que no exista la posibilidad de administrar ninguna unidad de sangre sin que previamente hubiera sido analizada $(5,8)$. Se observa, además, la necesidad de desarrollar y mejorar los procedimientos de promoción de la donación, selección de donantes, selección de pruebas con adecuada sensibilidad y especificidad, entre otros, con el fin de disminuir al máximo el riesgo de transmitir una infección vía transfusión.

\section{Agradecimientos}

Los autores expresan sus agradecimientos a los directores y coordinadores de bancos de sangre del país y a los coordinadores seccionales y distritales de bancos de sangre, por la excelente información suministrada; a la doctora Gloria Múnera por el apoyo en la traducción y al doctor Jorge Jara, jefe de División del Centro de Control de Enfermedades del INS por la revisión del texto. 


\section{Referencias}

1. Centers for Disease Control. Possible transfusion associated to acquired inmune deficiency syndrome AIDS. MMWR 1982;31:652-4.

2. Sepúlveda-Amor J, et al. La estrategia de prevención de la tranmisión del VIH/SIDA a través de la sangre y sus derivados en México. Salud Pública de México 1995; 37:624-35.

3. República de Colombia. Ministerio de Salud, Decreto 1571; agosto 1993.

4. Centers for Disease Control. Public Health Service guidelines for testing and counseling blood and plasma donors for human inmunodeficiency virus type 1 antigen. MMWR 1996;45:RR-2.
5. Organización Panamericana de la Salud. Manual de procedimientos de control de calidad para los laboratorios de serología de los bancos de sangre. Primera ed. Washington, D.C.: OPS; 1994:5-8.

7. Boral LI, Henry JB. Transfusion medicine. In: Henry JB, ed. Clinical diagnosis and management by laboratory methods. Philadelphia: Saunders; 1996:793-801.

8. Surgenor DM, Wallace EL, Hao SHS, Chapman RH. Collection and transfusion of blood in the United States, 1982-1988. N Engl J Med 1990;322:1646-51.

9. Lacritz EM, Satten GA, Aberle-Grasse J et al. Estimated risk of transmission of the human inmunodeficiency virus by screened blood in the United States. N Engl J Med 1995;333:1721-5. 